\title{
Tests of Improved Kogut-Susskind Fermion Actions
}

\author{
K. Orginos and D. Toussaint ${ }^{\mathrm{a} *}$ \\ ${ }^{a}$ Department of Physics, University of Arizona, Tucson, AZ 85721, USA
}

Improved Kogut-Susskind quark actions containing repeatedly smeared links are studied to address the issue of flavor symmetry restoration. As a measure of the flavor symmetry restoration the mass spectrum of all the pions is computed. We present results for several variants of the "fat" actions that are suitable for full QCD simulations.

\section{INTRODUCTION}

Improvements of the Kogut-Susskind quark action may allow extraction of continuum physics from coarser lattices than would be required with the simplest formulation. This is especially important for full QCD simulations, which are much more time consuming than quenched calculations. Improvement of the rotational symmetry and the dispersion relation can be achieved by introducing the Naik term, a coupling to third nearest neighbors. A more severe problem is flavor symmetry breaking, which is large for the currently accessible lattice spacings. It has been shown that smearing of the gauge links reduces flavor symmetry breaking. This is because smearing reduces the coupling to high transverse momentum gluons which cause transitions among the corners of the Brillouin zone. There are various smearings proposed so far. The simplest one, which has been studied by the MILC collaboration [1], is the introduction of the 3-link staple to the coupling of the nearest neighbors. A more extensive smearing has been studied by Sinclair and Lagae [2], who have shown that flavor symmetry breaking is further reduced. Finally, extensive APE smearing has been studied in $\mathrm{SU}(2)$ quenched spectroscopy [3], showing degeneracy within statistical errors between the Goldstone pion and the local non-Goldstone pion $\left(\pi_{2}\right)$.

In this paper, we study the effects of several types of smearing on the flavor symmetry break-

\footnotetext{
*Presented by D. Toussaint. Supported by the US DOE. Computations were done at ORNL and NERSC as part of the MILC collaboration.
}

ing, extending the results reported in Ref. [4]. Our goal is to find an action that achieves small flavor symmetry breaking, yet is localized enough to allow for a relatively cheap force computation when used in dynamical simulations. As a measure of flavor symmetry breaking we use the mass splittings of all the sixteen pions (in eight separate representations of the lattice symmetry group) [5] in hadron spectroscopy on a common set of stored lattices.

\section{ACTIONS TESTED}

As a guide in the construction of actions with improved flavor symmetry breaking we require that the coupling of the quarks to high transverse momentum gluons is minimized. Consider an action which has links smeared by a 3-link staple $S^{(3)}$, a 5-link staple $S^{(5)}$ and a 7-link staple $S^{(7)}$ :

$$
\begin{aligned}
U_{\mu}(x) & \rightarrow c_{1} U_{\mu}(x)+\sum_{\nu}\left[w_{3} S_{\mu \nu}^{(3)}(x)+\right. \\
& \left.+\sum_{\rho}\left(w_{5} S_{\mu \nu \rho}^{(5)}(x)+\sum_{\sigma} w_{7} S_{\mu \nu \rho \sigma}^{(7)}(x)\right)\right] \\
S_{\mu \nu}^{(3)}(x) & =U_{\nu}(x) U_{\mu}(x+\hat{\nu}) U_{\nu}^{\dagger}(x+\hat{\mu}) \\
S_{\mu \nu \rho}^{(5)}(x) & =U_{\nu}(x) S_{\mu \rho}^{(3)}(x+\hat{\nu}) U_{\nu}^{\dagger}(x+\hat{\mu}) \\
S_{\mu \nu \rho \sigma}^{(7)}(x) & =U_{\nu}(x) S_{\mu \rho \sigma}^{(5)}(x+\hat{\nu}) U_{\nu}^{\dagger}(x+\hat{\mu})
\end{aligned}
$$

In the weak coupling limit the couplings $V_{1}, V_{2}$, and $V_{3}$ to the gauge field with one, two or three of the transverse momentum components $\pm \pi / a$ can be written as functions of the staple couplings $w_{3}, w_{5}, w_{7}$, and the single link coupling $c_{1}$ :

$V_{1}=c_{1}+2 w_{3}-8 w_{5}-48 w_{7}$ 
$V_{2}=c_{1}-2 w_{3}-8 w_{5}+48 w_{7}$

$V_{3}=c_{1}-6 w_{3}+24 w_{5}-48 w_{7}$

The overall normalization condition

$c_{1}+6 w_{3}+24 w_{5}+48 w_{7}=1$

is used to ensure that the total coupling to the nearest neighbor in the free field limit is one. For $c_{1}=2 w_{3}=8 w_{5}=48 w_{7}=1 / 8$, all the couplings to gluons with any of the transverse momenta equal to $\pm \pi$ are zero. This set of parameters defines our "Fat7" action. The "Fat5" action is constructed by requiring that the magnitude of all the couplings $V$ is minimized. The "Fat5" couplings are $c_{1}=2 w_{3}=8 w_{5}=1 / 7, w_{7}=0$, which give $\left|V_{1}\right|=\left|V_{2}\right|=\left|V_{3}\right|=1 / 7$.

We have also tested an action ("All5") which contains all the non self-intersecting length 5 paths that connect nearest neighbors and third nearest neighbors. At the free field limit, such an action is the same as the "Fat5" action with a Naik term. The paths connecting nearest neighbors divide into three classes. The planar paths that displace the fundamental link by 0 or 2 sites with total weight $c_{1}$, the planar paths that displace the fundamental link by 1 site with total weight $w_{3}$, and the non-planar paths with total weight $w_{5}$. If we use the "Fat5" parameters, appropriately scaled to accommodate the Naik term, and distribute the weight equally among the members of each class of paths, the couplings $V$ are minimized.

Together with the MILC collaboration, Anna Hasenfratz and Chet Nieter, this work is now being extended to include "APE smeared" actions, where the fattened link is projected back on to $\mathrm{SU}(3)$. Here we present two preliminary results. "Ape1" has one level of APE smearing with APE parameter $\alpha=0.75$ [3], which was chosen to match the MILC fat action. The MILC fat action and the "Ape1" action differ only by the projection to $\mathrm{SU}(3)$. The second variation of APE smeared action we tested is "Ape4", which has four APE smearings with $\alpha=0.5$.

\section{SIMULATIONS AND RESULTS}

For our spectroscopy, we used lattices with dynamical Kogut-Susskind quarks. These lat- tices were produced with the Symanzik improved gauge action (same lattices as those in 顿). The dynamical fermion action used was the MILC "fat Naik action" with Dirac matrix $2 m+\not D$, where

$$
\begin{gathered}
\not D(x, y)=\sum_{\mu=-4,4} \eta_{\mu}(x) \operatorname{sign}(\mu) \times[ \\
\left(c_{1} U_{\mu}(x)+w_{3} \sum_{\nu \neq \mu} S_{\mu \nu}^{(3)}(x)\right) \delta_{y, x+\hat{\mu}} \\
\left.+c_{3} U_{\mu}(x) U_{\mu}(x+\hat{\mu}) U_{\mu}(x+2 \hat{\mu}) \delta_{y, x+3 \hat{\mu}}\right] .
\end{gathered}
$$

Here, $c_{1}=(9 / 8)(1 / 4)$ is the coefficient of the conventional single-link term, $c_{3}=-1 / 24$ is the coefficient of the third nearest neighbor (Naik) term, and $w_{3}=(9 / 8)(1 / 8)$ is the coefficient of the staple term.

Spectroscopy was done on $12^{3} \times 32$ lattices with $\beta_{i m p}=7.3$ and $16^{3} \times 48$ lattices with $\beta_{i m p}=7.5$. At $\beta_{i m p}=7.3$ we used $m=0.02$ and 0.04 , while at $\beta_{i m p}=7.5$ we used $m=0.015$ and 0.030 . In order to have a fair comparison of the actions tested, for each valence action we did spectroscopy on the same set of lattices and interpolated the spectrum of all the sixteen pions to the quark mass where $M_{G} / M_{\rho}=0.55\left(M_{G}\right.$ is the Goldstone pion mass). Successive lattices were separated by five molecular dynamics time units, and sample sizes ranged from 48 to 60 lattices.

In Fig. 1 and Fig. 2 we present the spectrum of all the sixteen pions interpolated to $M_{G} / M_{\rho}=$ 0.55 , for $\beta=7.3$ and $\beta=7.5$ respectively. We present data for the actions we tested and for comparison we also plot the data for the standard one link (OL) Kogut-Susskind action and the "fat Naik action" (OFN). The lowest level is always the Goldstone pion at 0.55 . The highest is the 3-link pion $\gamma_{0} \gamma_{5} \otimes \mathbf{1}$. The intermediate levels come in nearly degenerate pairs. In order to distinguish them, they are plotted shifted left and right from the center. The second level is the $\pi_{2}$, $\gamma_{0} \gamma_{5} \otimes \gamma_{0} \gamma_{5}$ (left) and the 1-link $\gamma_{5} \otimes \gamma_{i} \gamma_{5}$ (right). The third is the 2-link $\gamma_{5} \otimes \gamma_{i} \gamma_{0}$ (left) and the 1link $\gamma_{0} \gamma_{5} \otimes \gamma_{i} \gamma_{j}$ (right). The fourth is the 3 -link $\gamma_{5} \otimes \gamma_{0}$ (left) and the 2 -link $\gamma_{0} \gamma_{5} \otimes \gamma_{i}$ (right). The above degeneracies are predicted in the contribution [7] by Lee and Sharpe in this conference. 


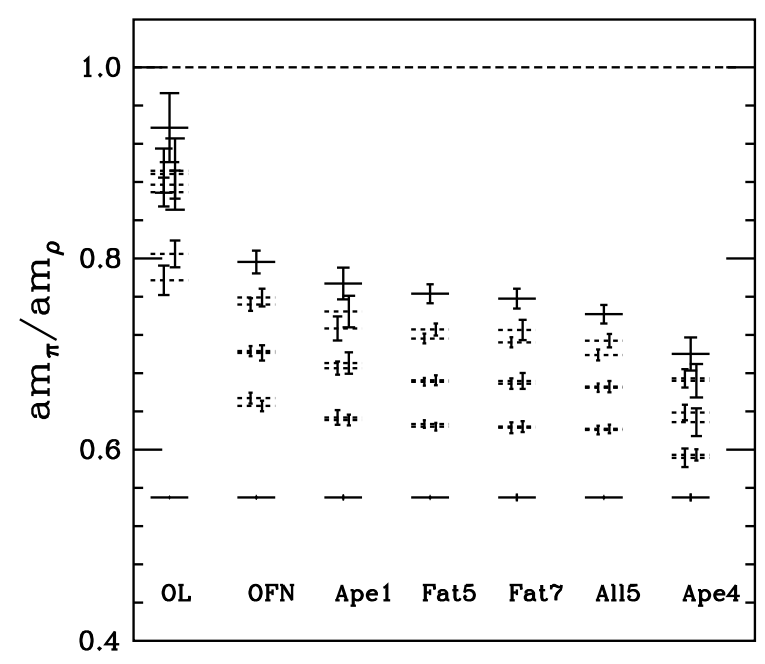

Figure 1. Interpolated masses for $\beta_{i m p}=7.3$.

From our data it can be seen that in general, smearing improves flavor symmetry breaking. Of the actions tested here, flavor symmetry breaking is smallest with the "Ape4" action, which is also the one with the most extensive smearing. Unfortunately, such an action is very costly for dynamical simulations. "Ape1" seems better than the OFN action. This leads us to conclude that the projection to SU(3), which APE smearing does, contributes to the improvement of the flavor symmetry breaking. The "Fat5" action gives some additional improvement over the "Ape1". It would be interesting to check if a projection of the "Fat5" link to SU(3) would give a further improvement. The "Fat7", which has all the couplings to gluons with transverse momenta $\pm \pi$, does not improve significantly over the "Fat5" action. Finally, "All5", which is the same as "Fat5" from the weak coupling point of view, does improve significantly over "Fat5". However, when the cost of the force computation is taken into account, the best of the above actions for full QCD calculations may be the "Fat5" (with the Naik term added) or even the "OFN" action. The decrease in flavor symmetry breaking as $\beta_{i m p}$ increases form 7.3 to 7.5 is consistent with the expected $a^{2}$ dependence of lattice artifacts with

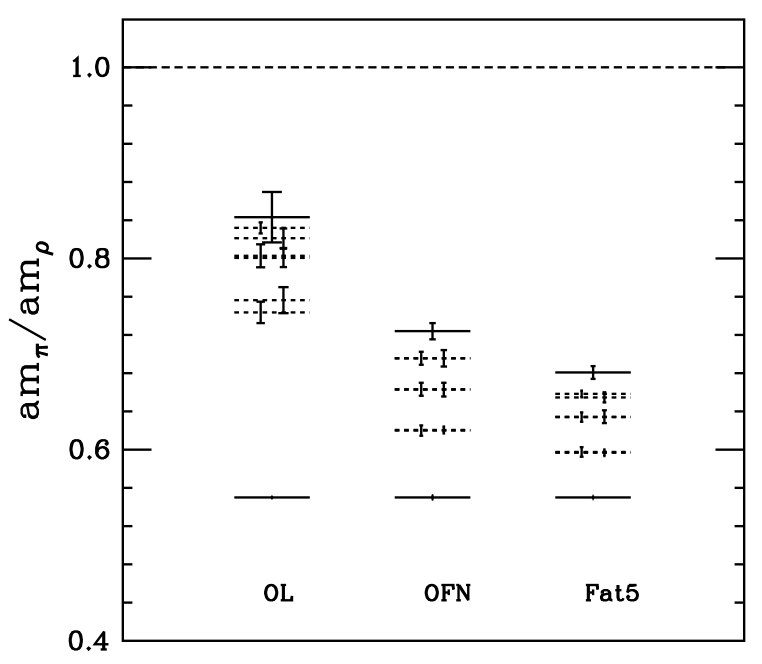

Figure 2. Interpolated masses for $\beta_{i m p}=7.5$.

Kogut-Susskind quarks.

Clearly, our data show that one has to look at the spectrum of all the sixteen pions before drawing any conclusions for the quality of the action. In particular, for studies of QCD thermodynamics with the strange quark [6], one would like to have all the pions light compared to the kaons. In order to do so, we have to go to lattice spacings significantly smaller [4] than those predicted from looking just at the local non-Goldstone pion.

\section{REFERENCES}

1. T. Blum et al., Phys. Rev. D 55, 1133 (1997).

2. J.F. Lagae, D.K. Sinclair, hep-lat/9806014; Nucl. Phys. (Proc. Suppl.) 63, 892 (1998).

3. T. DeGrand, A. Hasenfratz and T. Kovacs, Phys. Lett. 420B, 97 (1998).

4. K. Orginos, D. Toussaint hep-lat/9805009.

5. M.F.L. Golterman, Nucl. Phys. B 273, 663 (1986).

6. J.B. Kogut and D.K. Sinclair, Phys. Lett. B229, 107 (1989); F.R. Brown et al., Phys. Rev. Lett. 65, 2491 (1990); Y. Iwasaki et al., Phys. Rev. D 54, 7010 (1996).

7. W. Lee, S. Sharpe (in this proceedings) heplat/9809026. 